\title{
Atomic Observation on Alternating Heteroepitaxial Nanostructures in Na-ion
}

\section{Layered Oxide Cathodes}

Sheng $\mathrm{Xu}^{1}$, Huang Qun ${ }^{2}$, Jiatu Liu ${ }^{1,2}$, Weifeng $\mathrm{Wei}^{2}$ and Peng Wang ${ }^{1}$

1. National Laboratory of Solid State Microstructures, College of Engineering and Applied Sciences and Collaborative, Innovation Center of Advanced Microstructures, Nanjing University, Nanjing 210093, P. R. China.

2. State Key Laboratory of Powder Metallurgy, Central South University, Changsha, Hunan 410083, P. R. China.

Li-ion batteries (LIBs) has been used as large energy storage device over decades. Due to the increasing costs and limited Li resources, Na-ion batteries (NIBs) with similar storage mechanisms to LIBs are potentially able to be the next generation electrochemical energy storage device [1]. Layered oxide compounds with the general formula of $\mathrm{Na}_{\mathrm{x}} \mathrm{TMO}_{2}(\mathrm{TM}=\mathrm{Mn}, \mathrm{Co}$, $\mathrm{Ni}$, etc.) can provide two dimensional (2D) diffusion path for $\mathrm{Na}$ intercalation/deintercalation [2,3]. However, NIBs suffer from the low capacity and cyclability, and people are devoting to solve these problems [4]. Here we report a novel layered NIBs cathode, which combine the $\mathrm{Li}_{2} \mathrm{MnO}_{3}$ with the Na-P3 phase. This heteroepitaxial nanostructure cathode can improve the rate performance and higher initial capacity.

By the high-resolution scanning transmission electron microscopy (STEM) combined with energy X-ray dispersive spectroscopy (EDS), we can clearly distinguish the different elements. A double-aberration corrected FEI Titan G2 60-300 STEM, operated at $300 \mathrm{keV}$, was used. The screen current was $40 \mathrm{pA}$, and the convergent angle was $20.5 \mathrm{mrad}$. The EDS was acquiring using the Bruker Super- $\mathrm{X}^{\mathrm{TM}}$ technology with four detectors. To see the Li distributions, the electron energy loss spectroscopy (EELS) was recorded using a Gatan Imaging Filter (GIF) spectrometer attached to the microscope.

Figure 1.a shows the elemental mapping of $\mathrm{Na}-\mathrm{P} 3 / \mathrm{Li}_{2} \mathrm{MnO}_{3}$ cathodes, which can be distinguished by the contrast of concentration of different elements. It can be clearly see the Na-P3 phase in elements mapping of $\mathrm{Co}, \mathrm{Ni}$ and $\mathrm{Na}$ in brighter contrast, and the $\mathrm{Mn}$ element are gathering in $\mathrm{Li}_{2} \mathrm{MnO}_{3}$, which has lower contrast in HAADF images. To confirm the Li distributions, Figure 1.b shows the Li K-edge mapping by EELS. The lower contrast area in STEM images, which represents the $\mathrm{Li}_{2} \mathrm{MnO}_{3}$, shows the aggregation area of Li elements. By STEM imaging with spectroscopy technologies, the alternating heteroepitaxial structure can be easily seen and this explains to the unique electronic performance of the $\mathrm{Na}-\mathrm{P} 3 / \mathrm{Li}_{2} \mathrm{MnO}_{3}$ cathode.

References:

[1] MH Han et al, Energ. Environ. Sci. 8 (2015), p. 81

[2] R Kataoka et al, J. Electrochem. Soc. 160 (2013), p. A933 
[3] E Lee et al, Adv. Energy. Mater. 4 (2014), p. 1400458

[4] N Yabuuchi et al, Chem. Rev. 114 (2014), p. 11636
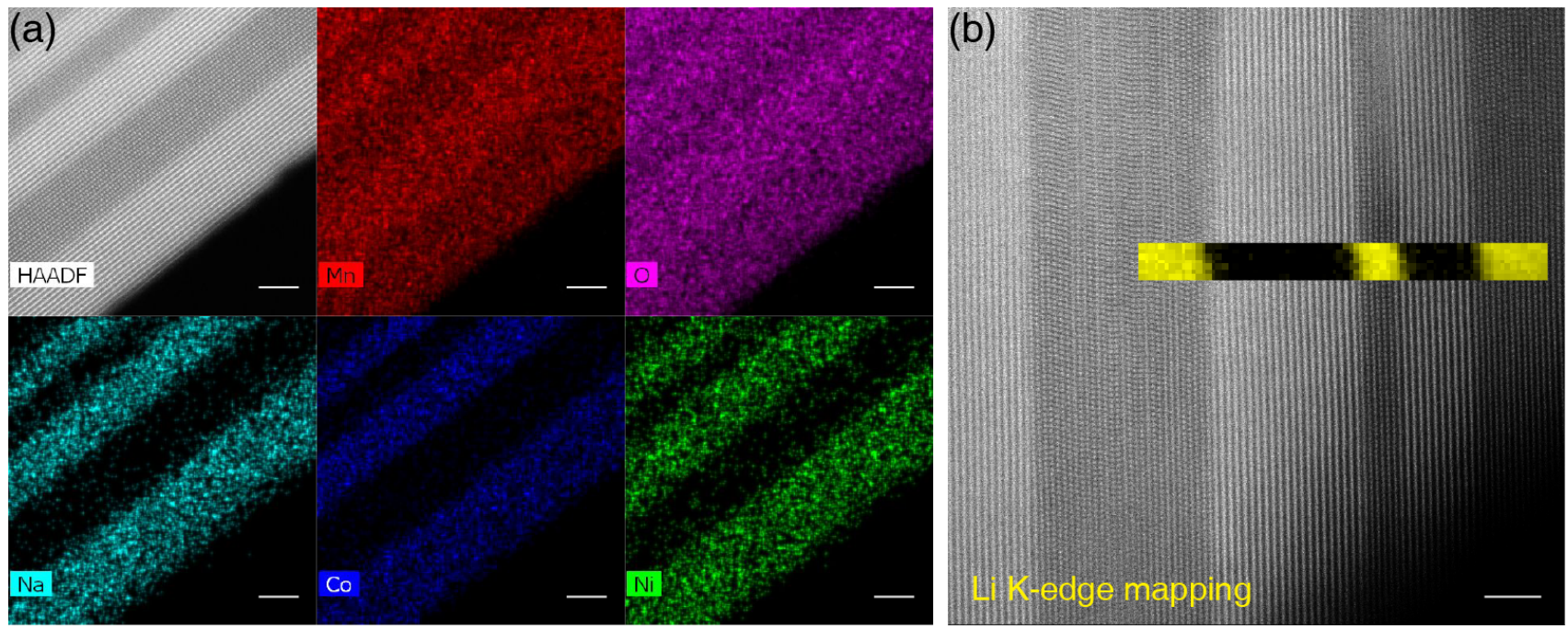

Figure 1. (a) STEM-EDS mapping, (b) EELS mapping of Li K-edge. The brighter contrast areas in STEM-image corresponds to the $\mathrm{Na}$, $\mathrm{Co}$ and $\mathrm{Ni}$ elements mapping, while the lower ones are connected with the $\mathrm{Mn}, \mathrm{O}$ and Li elements mapping. The scale bar is $5 \mathrm{~nm}$. 